\title{
Management guidelines for acute infective diarrhoea/ gastroenteritis in infants
}

\author{
D F Wittenberg
}

Background. Acute onset vomiting and diarrhoea is one of the most common illnesses of infancy, and is second only to respiratory illnesses as a cause of childhood deaths worldwide. Existing guidelines for management of diarrhoea are often ignored in public and private practice, possibly because of a perception that the guidelines are too simple, or because of expectations of the need to give 'real' drug therapy to stop diarrhoea.

Objectives. This guideline provides a problem-based approach to the basics of present-day management of acute gastroenteritis, and discusses the evidence for the recommendations.

Recommendations. Each episode of diarrhoea must be seen as an opportunity for caregiver education in the prevention of the illness, in the 'what' and 'how' of oral rehydration and re-feeding, and in the recognition of when to seek help. The vast majority of patients recover rapidly, but serious complications do occur and must be recognised and managed correctly.

Validation. The guidelines are endorsed by the Paediatric Management Group (PMG) in South Africa.

Conclusion. The aim of management is to help the child to maintain or regain hydration, and to recover from diarrhoea, with careful attention to adequate oral rehydration and judicious re-feeding.

S Afr Med J 2012;102:104-107.
Acute onset vomiting and diarrhoea is one of the most common illnesses of infancy. Caused by acute infection with a variety of viruses, bacteria and parasites, the condition is easily recognised by caregivers and is usually managed at home. Numerous traditional and folk remedies exist, owing to the common and well-recognised nature of the condition; these might have been tried before the child is brought for medical attention.

In this era of highly prevalent HIV infection, it must be remembered that each episode of acute infective diarrhoea may occur in a patient with unsuspected pre-existing HIV infection; each healthcare contact should be an opportunity to establish the child's HIV status for optimal management.

Acute diarrhoea has several risks and complications; it may lead to life-threatening dehydration and electrolyte disturbances. When diarrhoea is not halted, there is a risk of disturbed digestion and absorption of nutrients with nutritional deterioration. Worldwide, diarrhoea is second only to respiratory illnesses as a cause of childhood deaths. ${ }^{1}$

\section{Prevention}

Gastroenteritis is caused by intestinal infection owing to contamination of food, water or hands. All health professionals should ensure caregiver education in the following main principles of prevention:

- Full and exclusive breast-feeding on demand: this protects against intestinal infections $s^{2,3}$ and prevents exposure to environmental contamination. Thriving breast-fed babies under 6 months of age do not require water supplements, even in hot weather., ${ }^{4,5}$

- Full immunisation including rotavirus vaccines: the new rotavirus vaccines are safe and reduce the severity of infection

Department of Paediatrics and Child Health, School of Medicine, Faculty of Health Sciences, University of Pretoria

D F Wittenberg, MB ChB, MD, FCP (Paed) (SA) and prevent deaths, but they do not prevent all cases of rotavirus diarrhoea. ${ }^{6}$

- Provision of safe water for drinking and food preparation.

- Proper hand-washing hygiene after toilet use and before food preparation and feeding.

- Safe disposal of human and other waste.

\section{Prevention of diarrhoea}

Full and exclusive breast-feeding

Rotavirus vaccination of all babies

Evidence Level A

Proper hand-washing hygiene

Safe water

Safe disposal of waste

\section{Management guidelines: acute \\ diarrhoea}

In view of the frequency and importance of the condition, bestpractice guidelines for management of diarrhoea have been published by the World Health Organization (WHO), ${ }^{7,9}$ American Academy of Pediatrics (AAP), ${ }^{10}$ and European Society of Paediatric Gastroenterology, Hepatology and Nutrition (ESPGHAN), ${ }^{11}$ among others. The use of these guidelines has been described in numerous countries; ${ }^{12,13}$ however, they are often ignored in practice, more frequently than those for other paediatric conditions such as asthma. ${ }^{14,15}$ Reasons for this include doctors' unwillingness to be imposed upon, failure by doctors to keep pace with changing views on hydration and nutrition, a perception that the guidelines are too simple, and parental expectations of 'real' drug therapy to stop diarrhoea.

The HIV status of each child with diarrhoea must be ascertained.

\section{Acute gastroenteritis: what are the problems?}

Vomiting is largely attributed to local factors and poor gastric emptying, and should not be treated with antiemetic drugs. ${ }^{16}$ Cyclizine and prochlorperazine have not proven useful and may carry a higher risk of toxic side-effects in young infants, and in the 
presence of dehydration. Ondansetron is moderately effective, ${ }^{17}$ but the cost and quantity required for a clinically significant effect negates general recommendation.

Antiemetic drugs are not recommended

Evidence Level A

Abdominal pain is usually spasmodic due to disordered motility, or is associated with colitis in dysentery. Metoclopramide should be considered in severe pain, at a dose of $0.1 \mathrm{mg} / \mathrm{kg}$ of body mass to a maximum of $10 \mathrm{mg}$; a total daily dose of $0.5 \mathrm{mg} / \mathrm{kg}$ should not be exceeded. ${ }^{16}$ The extrapyramidal side-effects of metoclopramide in young infants should warrant caution. Frequent small oral sips of a clear hypotonic glucose-containing fluid correct ketosis and prevent gastric overdistension, in addition to contributing to rehydration. In early gastroenteritis, solids or foods with a high protein, fat or fibre content slow down gastric emptying and may aggravate vomiting. Substitution with a clear, hypotonic fluid may result in earlier cessation of vomiting.

Diarrhoea is the manifestation of secretion/absorption disturbance and disordered motility: a symptom of damage already done in the infected gut. Antidiarrhoeal medication is not advised. Antidiarrhoeal formulations aim to reduce intestinal motility, reduce secretion of water and electrolytes, and adsorb fluid and toxins, thereby reducing the number of stools seen in the diaper; however, none treat the cause of diarrhoea or actual pathology, and their use may be associated with more side-effects in young children than in adults. Furthermore, adsorbents (e.g. kaolin) hide the true extent of water loss, risking underestimation of the amount of fluid required for hydration in severe diarrhoea.

Antibiotic therapy is not indicated in the majority of cases caused by viral infection. ${ }^{18}$ In bacterial infection, antibiotic therapy generally does not shorten the length of diarrhoea, except when administered early in the case of dysentery (ciprofloxacin for 3 days is recommended). ${ }^{7}$ Rather, antibiotics are required to prevent or limit the spread of infection to others (e.g. early in cholera), and should be prescribed for evident parenteral infection (e.g. urinary tract infection or otitis media).

Probiotics have variable effect in specific circumstances, ${ }^{19,20}$ and are generally not required, but could be considered in diarrhoea associated with nosocomial infections or antibiotic use. Probiotics are sometimes added to commercial preparations of oral rehydration solutions (e.g. Hydrachoice). ${ }^{21}$

Vitamin and mineral supplementation is required where the patient's nutritional state or history suggests the possibility of deficiencies. Zinc acetate $(10-20 \mathrm{mg} /$ day for 2 weeks) reduces the duration of diarrhoea and recurrence risk in developing countries. ${ }^{22-28}$ To date, no comparable studies are available in developed countries; however, it is known that zinc has an effect on mucosal functions involved in absorption of water and rehydration. ${ }^{23,24}$

\section{Drugs for diarrhoea}

Antibiotics do not improve diarrhoea

Andiarrhoeal mixtures and drugs are not useful

Probiotics have variable effectiveness in specific

circumstances

Zinc acetate for 2 weeks reduces duration and recurrence risks

Evidence Level A

Vitamin and mineral supplementation

should be given for deficiencies
Dehydration is the main acute risk of acute gastroenteritis. Watery stools develop as a result of a secretion-absorption imbalance of the gut mucosa; however, glucose-linked sodium absorption with secondary water uptake is maintained even in secretory states like cholera. This is the basis of oral rehydration therapy (ORT). Hypotonic fluids with a sodium content of $45-65 \mathrm{mmol} / \mathrm{l}$ (e.g. Rehidrat, Sorol) allow more rapid rehydration than fluids with a higher sodium content, ${ }^{29}$ but hypertonic sugar-based drinks with little sodium (e.g. cola and sports drinks) may result in osmotic aggravation. A home-made salt-and-sugar solution (SSS) (half a teaspoon of salt plus 8 teaspoons of sugar per litre of water) is useful, but of unpredictably variable concentration, and is therefore best used in the early stages before dehydration has set in.

Dehydration occurs because fluid loss exceeds fluid intake. In general, signs of dehydration lag behind water loss. The best guide to the risk of developing dehydration is the frequency of watery stools: assuming a deficit of $10 \mathrm{ml} / \mathrm{kg}$ of body mass for each watery stool. This amount of fluid should be offered to the child in addition to usual fluid intakes.

Initially, the emphasis is on maintaining fluid intake despite vomiting, offering feeds according to tolerability. Small frequent liquid feeds are better tolerated than large volumes or solids. Feeds should be followed with clear hypotonic fluids such as SSS, or even dilute juices or mildly sweetened tea - as much as the child wants, in small quantities. Commercial rehydration fluids are also suitable. Taste may be an issue in the case of anorexia or vomiting; caregivers may need to be creative in adapting or hiding the taste of rehydration fluids.

\section{Prevent dehydration}

Small frequent liquid feeds should be given with added clear fluids such as salt-and-sugar solution, diluted juices or mildly sweetened tea, even when the child is vomiting.

Once the child shows signs of dehydration, ORT takes precedence over feeding. The clinical signs of dehydration are variable; ${ }^{30}$ the degree of dehydration is most accurately predicted with a combination of the observer's experience, ${ }^{31,32}$ the measured acute weight loss, and the presence of metabolic acidosis. ${ }^{33}$ Placed together, these allow an assessment of whether dehydration is mild, moderate or severe. In particular, capillary filling time must be noted: a capillary refill time $(\mathrm{CFT})>3$ seconds in a dehydrated child indicates the need for intravenous resuscitation in addition to rehydration.

\section{Intravenous fluids in dehydration}

Severely dehydrated and shocked patients require intravenous resuscitation. Intravenous fluids are required in the following cases (in all others, ORT should be preferred):

- $\quad$ Resuscitation from shock

- Dehydration with severe acidosis and prolonged capillary refill time

- Severe abdominal distension and ileus

- An altered level of consciousness

- Resistant vomiting despite appropriate oral fluid administration

- Deterioration or lack of improvement after 4 hours of adequate oral fluids 
Initially, an isotonic solution - such as Lactated Ringer's or Normal Saline - should be chosen for resuscitation. If the circulation has improved after 1 - 2 boluses of $20 \mathrm{ml} / \mathrm{kg}$, given rapidly, the fluid can be changed to a rehydration fluid such as half-strength Darrow's solution with $5 \%$ dextrose, at a rate of $10 \mathrm{ml} / \mathrm{kg} / \mathrm{h}$. If the circulation has not improved after the second bolus, a third bolus of $20 \mathrm{ml} / \mathrm{kg}$ should be started, with transfer to hospital for intensive monitoring and care.

Patients receiving intravenous rehydration must be reassessed regularly, as the fluid administration rate may require adjustment, up or down, depending on the rate of improvement and ongoing loss.

ORT achieves faster rehydration and is associated with fewer admissions than parenteral rehydration. ${ }^{34,35} \mathrm{~A}$ hypotonic solution is recommended with a $45-65 \mathrm{mmol} / \mathrm{l}$ sodium content, approximately $2 \%$ glucose $(111 \mathrm{mmol} / \mathrm{l})$, and potassium and bicarbonate or citrate for alkali replenishment. Commercial rehydration solutions comply with these recommendations.

Oral rehydration solutions, provided in small frequent sips by teaspoon at a rate of $15-25 \mathrm{ml} / \mathrm{kg} / \mathrm{h}$, avoid gastric distension and vomiting. Manuals such as the Integrated Management of Childhood Illnesses, ${ }^{8}$ the South African Essential Drugs List (Hospital Level, Paediatrics) and the WHO Manual on the Management of Diarrhoea, ${ }^{9}$ provide recommended volumes of solutions to be given per hour. Most children are able to rehydrate within a few hours before they are ready to commence with small feeds. In the face of ongoing diarrhoea, extra fluids must still be offered for thirst and prevention of further dehydration.

$$
\text { Oral rehydration therapy is recommended Evidence Level A }
$$

\section{Metabolic problems and electrolyte disturbances}

These occur because of the loss of sodium, bicarbonate and potassium associated with watery stools. The electrolyte status of patients with severe diarrhoeal dehydration, circulatory disturbances or metabolic acidosis should be ascertained.

- Metabolic acidosis is almost always present if the patient has significant dehydration, and is identified by more rapid deep respirations with a clear chest on auscultation. Unless metabolic acidosis is severe, sodium bicarbonate is usually not required, as rehydration allows for correction. Rehydration fluid contains alkali in the form of citrate or bicarbonate. A wide anion gap may suggest the possibility of severe ketosis, salicylate intoxication or lactic acidosis.

- Large amounts of potassium are lost in diarrhoeal stools. Metabolic acidosis is associated with further urinary potassium loss. In acidosis, a shift of intracellular potassium to the extracellular compartment results in a spurious elevation of the serum level, despite intracellular potassium loss. All children suffering from severe diarrhoea should receive oral potassium chloride: $125 \mathrm{mg} 8$-hourly if under 1 year of age, and $250 \mathrm{mg}$ 8-hourly if over 1 year of age, until dehydration and acidosis are corrected.

- Sodium disturbances occur frequently. Sodium content of the stool water varies from plasma-like in secretory diarrhoea (such as cholera), to very low in pure osmotic diarrhoea. In general, serum sodium is inversely related to the state of the intracellular water compartment, i.e. raised serum sodium reflects intracellular dehydration. Hypo- and hypernatraemia management should follow recommended guidelines; however, where ORT is possible, the process of rehydration usually allows metabolic homeostasis to be re-established.

- Blood glucose disturbances occur in severely ill young children as a result of glycogen depletion with lack of intake, or in association with the stress response of dehydration. Blood glucose estimation should be performed by finger-prick test in all dehydrated children.

Dehydrated or malnourished children are likely to have a metabolic disturbance.

\section{Feeding during diarrhoea}

Intestinal infection does affect the digestive and absorptive function of the gut, and diarrhoea is a symptom of this malfunction. The degree and extent of mucosal damage is influenced by: ${ }^{36}$

- Age - infants under 3-6 months of age may be expected to have more severe intestinal injury from gut infection.

- Type and site of infection - viral diarrhoea involves the mucosal villi and can be expected to affect digestion and absorption, while toxigenic diarrhoea (e.g. cholera) does not affect the mucosal structure. Infection in the upper small gut affects digestion and absorption more than that in the colon.

- Pre-existing medical condition that affects the patient's recovery (e.g. HIV).

- Pre-existing nutritional state and lack of breast-feeding malnutrition results in predisposition to mucosal atrophy, and superimposed gut infection increases the risk of maldigestion (e.g. lactose intolerance).

The state of nutrition should be assessed in each child with diarrhoea.

In a well-nourished child, infection-induced mucosal damage may recover rapidly without a need for modified feeds. ${ }^{37,38}$ Once rehydrated, the child's usual feeds should be reintroduced. At first, it may be necessary to give smaller volumes more frequently to avoid vomiting; the diarrhoea may continue for a short while. Initially, the child may be unable to take full feeds and may lose weight, but the aim is to achieve full-volume feeding within $1-2$ days. There is no need to dilute or otherwise modify the usual feeds, provided that they are tolerated.

There is no need for feed change

in the usual case.

Evidence Level A

Breast milk is a hypotonic fluid that can be utilised simultaneously for hydration maintenance and feeding; breast-feeding should continue and even be increased during diarrhoea. Other milk formulas should not be used to hydrate the patient because of the high solute load, but after recovery from diarrhoea, extra food should be offered for nutritional recovery.

In malnourished children, acute gastroenteritis may be more severe, and recovery may be delayed. Greater vigilance is needed in suspecting possible maldigestion and malabsorption. Children should be monitored until full recovery from diarrhoea and resumption of weight gain

Persisting diarrhoea (longer than 2 weeks after acute onset diarrhoea) is a more serious condition; it is associated with nutritional 
deterioration and much of the mortality from diarrhoea. ${ }^{39,40}$ Small intestinal mucosal injury or bacterial overgrowth should be suspected and diagnosed by appropriate means if: (i) the persisting diarrhoea is associated with weight loss and a continued need for rehydration fluids; (ii) the child is under 6 months of age or is malnourished, or (iii) a complication such as lactose intolerance exists. Feed modification and substitution is usually required in a planned stepwise progression.

Diarrhoea that persists for longer than 2 weeks is no longer 'gastro. Persisting diarrhoea is associated with deterioration of nutritional state, and must be managed actively to enable digestive and nutritional recovery.

\section{Caregiver education}

Acute diarrhoea is predominantly a problem of fluids and feeding both being heavily dependent on the caregiver's understanding and reactions.

It is vital that caregivers understand the 'what' and the 'how' of ORT and re-feeding, and are given guidance on the need to seek further help in the event of the following:

- Ongoing vomiting despite small fluid sips, especially if associated with abdominal distension or pain

- $\quad$ Persisting fever after 24 hours of ORT

- Increasing lethargy and failure to feed

- Deteriorating hydration and failure to pass urine

- Presence of blood in the stools

- Diarrhoea persisting for more than 1 week.

\section{Conclusion}

The aim of management is to help the child to maintain or regain hydration, and to recover from diarrhoea. With careful attention to adequate oral rehydration and judicious re-feeding, acute infective diarrhoea should not be a frightening condition to caregivers.

Endorsement. The guidelines are endorsed for publication by the Paediatric Management Group (PMG) in South Africa.

\section{References}

1. Boschi-Pinto C, Velebit L, Shibuya K. Estimating child mortality due to diarrhoea in developing countries. Bull World Health Organ 2008;86(9):710-717.

2. Quigley MA, Kelly YJ, Sacker A. Breast-feeding and hospitalization for diarrhoeal and respiratory infection in the United Kingdom Millenium Cohort Study. Pediatrics 2007;119(4):e837-842.

3. Quigley MA, Cumberland P, Cowden JM, Rodriguez LC. How protective is breast-feeding against diarrhoeal disease in infants in 1990's England? A case-control study. Arch Dis Child 2006;91(3):245250

Satyanarayana L, Kumar S. Water supplementation in exclusively breastfed infants during summer in the tropics. Lancet 1991;337:929-933.

Williams HG. 'And not a drop to drink' - why water is harmful for newborns. Breastfeed Rev 2006;14(2):5-9.

6. Patel M, Pedreira C, de Olivieria LH, et al. Association between pentavalent rotavirus vaccine and severe rotavirus diarrhoea among children in Nicaragua. JAMA 2009;301(2):2243-2251.

7. World Health Organization/United Nations Children's Fund. Clinical management of acute diarrhoea. Geneva: World Health Organization, 2004. http://www.who.int/child_adolescent_health/documents/ who_fch_cah_04_7/en/index.html (accessed 31 October 2011).
8. World Health Organization/United Nations Children's Fund. Handbook: Integrated Management of Childhood Illness (IMCI), 2005 Technical Update. Geneva: World Health Organization, 2005.

9. World Health Organization. Implementing the New Recommendations on the Clinical Management of Diarrhoea: Guidelines for Policy Makers and Programme Managers. Geneva, World Health Organization, 2006

10. American Academy of Pediatrics. Practice parameter: the management of acute gastroenteritis in young children. Pediatrics 1996;97(3):424-435

11. Sandhu BK. Practical Guidelines for the Management of Gastroenteritis in Children. J Pediat Gastroenterol Nutr 2001;33:S36-S39.

12. Armon K, Stephenson T, MacFaul R, Eccleston P, Werneke U. An evidence and consensus based guideline for acute diarrhoea management. Arch Dis Child 2001;85(2):132-141.

3. Jones SA Clinical pathway for pediatric gastroenteritis. Gastroenterol Nurs 2003;26(1):7-18.

14. Hoekstra JH. Acute gastroenteritis in industrialized countries: compliance with guidelines for treatment. J Pediatr Gastroenterol Nutr 2001;33:S31-S35.

5. Migowa AN, Gatim B, Nduati R. Adherence to oral rehydration therapy among inpatient children aged 1-59 months with some or no dehydration. J Trop Pediatr 2010;56(2):103-107.

16. DeCamp LR, Byerley JS, Doshi N, et al. Use of antiemetic agents in acute gastroenteritis: a systematic review and myerles

17. Levine DA. Antiemetics for acute gastroenteritis in children. Curr Opin Pediatr 2009;21:294-298.

18. Abba K, Sinfield R, Hart CA, Garner P. Antimicrobial drugs for persistent diarrhoea of unknown or non-specific cause in children under six in low and middle income countries: systematic review of non-specific cause in children under six in low and mi

19. Szajewska $\mathrm{H}$, Mrukowicz JZ. Probiotics in the treatment and prevention of acute infectious diarrhea in infants and children: a systematic review of published randomized, double-blind, placebo-controlled trials. J Pediatr Gastroenterol Nutr 2001;33(S2):S17-25.

20. Guandalini S, Pensabene L, Abu-Zikri M, et al. Lactobacillus GG administered in oral rehydration solution in children with acute diarrhoea: a multi-centre European trial. J Pediatr Gastroenterol Nutr 2000;30:54-60.

21. Passariello A, Terrin G, de Marco G, et al. Efficacy of new hypotonic oral rehydration solution containing zinc and probiotics in the treatment of childhood acute diarrhoea: a randomised controlled trial. J Pediatr 2011;158(2):288-292.el.

22. Scrimgeour AG, Lukaski HC. Zinc and diarrheal disease: current status and future perspectives. Curr Opin Clin Nutr Metab Care 2008,11:711-717.

23. Bhutta Z, Bird S, Black RE, et al. Therapeutic effects of oral zinc in acute and persistent diarrhea in children in developing countries: pooled analysis of randomized controlled trials. Am J Clin Nutr 2000;72:1516-1522.

24. Scrimgeour AG, Condlin ML. Zinc and micronutrient combinations to combat gastrointestinal inflammation. Curr Opin Clin Nutr Metab Care 2009,12:653-660.

25. Awathi S. Zinc supplementation in acute diarrhea is acceptable, does not interfere with oral rehydration, and reduces the use of other medications: a randomized trial in five countries. J Pediat Gastroenterol Nutr 2006;42:300-305.

26. Canani RB, Ruotolo S. The dawning of the 'Zinc Era' in the treatment of pediatric acute gastroenteritis worldwide? J Pediatr Gastroenterol Nutr 2006;42:253-255.

7. Lazzerini M, Ronfani L. Oral zinc for treating diarrhoea in children. Cochrane Database Syst Rev 2008;(3):CD005436.

28. Patro B, Golicki D, Szajewska H. Meta-analysis: zinc supplementation for acute gastroenteritis in children. Aliment Pharmacol Ther 2008;28(6):713-723.

29. Santosham M, Fayad I Abu Zikri M, et al. A double blind clinical trial comparing WHO or rehydration solution with a reduced osmolarity solution containing equal amounts of sodium and glucose. J Pediatr 1996;128(1):45-51.

30. Mackenzie A, Barnes G, Shann F. Clinical signs of dehydration in children. Lancet 1989;2:605-607.

31. Duggan C, Refat M, Hashem M, et al. How valid are clinical signs of dehydration in infants. J Pediat Gastroenterol Nutr 1996;22(1):56-61

32. Roland D, Clarke C, Borland ML, Pascoe EM. Does a standardised scoring system of clinical sign reduce variability between doctors' assessments of the potentially dehydrated child? J Paediatr Child Health 2010;46(3):103-107.

33. Colletti JE, Brown KM, Sharieff GQ, Barata IA, Ishimine P. The management of children with gastroenteritis and dehydration in the emergency department. J Emerg Med 2010;38(5):686-698.

34. Hartling L, Bellemare S, Wiebe N, et al. Oral versus intravenous rehydration for treating dehydration due to gastroenteritis in children. Cochrane Database Syst Rev 2006;3:CD004390.

35. Spandorfer PR, Alessandrini EA, Joffe MD, Localio R, Shaw KN. Oral versus intravenous rehydration of moderately dehydrated children: a randomized controlled trial. Pediatrics 2005;115:295-301.

36. Gracey M. Nutritional effects and management of diarrhea in infancy. Acta Paediatr Suppl 1999;88(430):110-126

37. Walker-Smith J, Sandhu BK, Isolauri E, et al. Recommendations for feeding in childhood gastroenteritis. Medical position paper on behalf of ESPGAN. J Pediatr Gastroenterol Nutr 1997;24:619-620.

38. Sandhu $\mathrm{BK}$, Isolauri $\mathrm{E}$, Walker-Smith $\mathrm{J}$, et al. Early feeding in childhood gastroenteritis. A multicenter study on behalf of the European Society of Paediatric Gastroenterology and Nutrition Working Group on Acute Diarrhoea. J Pediatr Gastroenterol Nutr 1997:24:522-527.

39. Teka T, Faruque AG, Fuchs GJ. Risk Factors for death in under-age-five children attending a diarrhoe treatment centre. Acta Paediatr 1996;85(9):1070-1075.

40. Victora CG, Huttly SR, Fuchs GJ, et al. International differences in clinical patterns of diarrhoeal deaths : a comparison of children from Brazil, Senegal, Bangladesh and India. J Diarrhoeal Dis Res 1993;11(1):25-29.

Accepted 31 October 2011. 